\title{
Sensing Single Molecules with Carbon-Boron-Nitride Nanotubes
}

\begin{tabular}{|r|l|}
\hline Journal: & Journal of Materials Chemistry C \\
\hline Manuscript ID: & TC-ART-07-2015-002284 \\
\hline Datticle Type: & Paper \\
\hline Complete List of Authors: & $\begin{array}{l}\text { Algharagholy, laith; Lancaster University, Physics } \\
\text { Pope, Thomas; Catania University, Physics } \\
\text { Al-Galiby, Qusiy; Lancaster University, Physics } \\
\text { Sadeghi, Hatef; Lancaster University, Physics } \\
\text { Bailey, Steven; Lancaster University, Physics } \\
\text { Lambert, Colin; Lancaster University, Physics }\end{array}$ \\
\hline
\end{tabular}

\section{SCHOLARONE ${ }^{\text {m }}$ \\ Manuscripts}




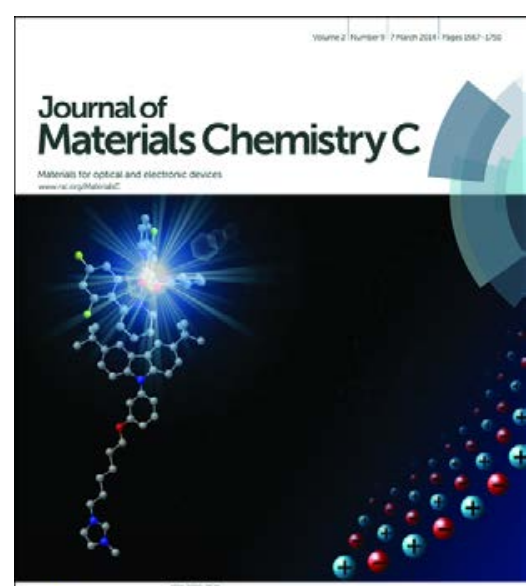

\section{Journal of Materials Chemistry C} Materials for Optical, Magnetic and Electronic Devices

\section{Full paper submission}

Journal of Materials Chemistry $C$ is a weekly journal in the materials field. The journal is interdisciplinary, publishing work of international significance on all aspects of materials chemistry related to optical, magnetic and electronic devices. Articles cover the fabrication, properties and applications of materials.

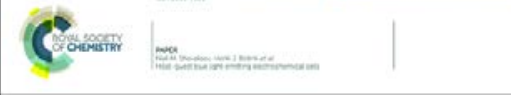

2013 Impact Factor of Journal of Materials Chemistry: $\mathbf{6 . 6 2 6}$

For more information go to www.rsc.org/materialsC

The following paper has been submitted to Journal of Materials Chemistry $C$ for consideration as a full paper.

Journal of Materials Chemistry $C$ wishes to publish original research that demonstrates novelty and advance, either in the chemistry used to produce materials or in the properties/applications of the materials produced. Work submitted that is outside of these criteria will not usually be considered for publication. The materials should also be related to the theme of materials for optics, magnetics and electronics.

Routine or incremental work, however competently researched and reported, should not be recommended for publication if it does not meet our expectations with regard to novelty and impact.

It is the responsibility of authors to provide fully convincing evidence for the homogeneity and identity of all compounds they claim as new. Evidence of both purity and identity is required to establish that the properties and constants reported are those of the compound with the new structure claimed.

Thank you for your effort in reviewing this submission. It is only through the continued service of referees that we can maintain both the high quality of the publication and the rapid response times to authors. We would greatly appreciate if you could review this paper in two weeks. Please let us know if that will not be possible.

Once again, we appreciate your time in serving as a reviewer. To acknowledge this, the Royal Society of Chemistry offers a $\mathbf{2 5 \%}$ discount on its books: http://www.rsc.org/Shop/books/discounts.asp. Please also consider submitting your next manuscript to Journal of Materials Chemistry $C$.

Best wishes,

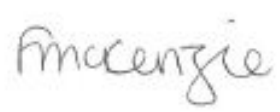

Dr Fiona McKenzie

Executive Editor, Journal of Materials Chemistry C 
$24 / 7 / 2015$

Dear Reviewer,

It is a pleasure to submit our manuscript entitled "Sensing Single Molecules with Carbon-BoronNitride Nanotubes," for publication in Journal of Materials Chemistry C.

Molecular recognition is the basis of fundamental biological processes such as transcription and translation of genetic information. Considerable effort has been devoted to design of receptors or sensors that recognise and sense target analytes. Typically this involves designing a receptor, which selectively binds most strongly to a particular analyte. Label-free methods for detecting small molecules are a desirable target technology, because they avoid the need for chemical modification or separation of the analytes, potentially leading to lower costs. Examples of label-free detection include micelle-based bacterial quorum sensing [1] aptamer-based sensing platforms [2], label-free, sequence specific DNA sensing based on fluorescence resonant energy transfer (FRET) [3] and nuclear magnetic resonance [4], nanoplasmonics [5], and surface enhanced Raman spectroscopy (SERS) [6]. However all of these require expensive detectors and are not shrinkable to sub-micronscale devices and therefore the cost-lowering advantages of label-free sensing are not yet fully realised.

In our paper, we demonstrate a completely different strategy for molecular recognition based on electrical junctions formed from carbon nanotube (CNT) electrodes, separated by a boron nitride (BN) spacer. The electrical conductance of such junctions changes when an analyte binds to the BN region and the response to analyte binding depends on the length of the $\mathrm{BN}$ region. Crucially the collection of responses from junctions of different $\mathrm{BN}$ lengths constitutes a unique fingerprint that discriminates between different analytes.

The rewards for realising cheap and versatile discriminating sensors cannot be overstated. During the next three years, the market for chemical sensing with applications to personal health, mood sensing, context-aware computing and the deployment of sensory swarms is expected to grow exponentially, provided low-power, low-cost discriminating molecular sensors can be developed.

Our work will be of interest to the molecular electronics and nano electronics communities, as well as chemists and physicist working on the fundamental aspects of functionalised surfaces, smart functional packaging, enhanced dyes, pigments and fluorophores. We are confident our work meets the criteria and standards for publishing in Journal of Materials Chemistry C.

Yours sincerely,

Prof Colin Lambert

[1]Davis, B., J. Richens, and P. O'Shea, Biophysical journal, 2011. 101(1): p. 245-254.

[2] Baker, B.R., et al., JACS, 2006. 128(10): p. 3138-3139.

[3] Liu, Z., H.-L. Wang, and M. Cotlet, Chemistry of Materials, 2014. 26(9): p. 2900-2906.

[4] Garimella, P.D., et al., JACS, 2013. 136(1): p. 164-168.

[5] Im, H., et al., Nature Biotechnology, 201432 p. 490.

[6] Li, M., et al., J. Biomedical optics, 2014. 19(5): p. 50501-50501. 


\title{
Journal of Materials Chemistry C
}

\section{Sensing Single Molecules with Carbon-Boron-Nitride Nanotubes}

Received 00th January 20xx, Accepted 00th January 20xx

DOI: $10.1039 / \times 0 \times x 00000 x$

www.rsc.org/

\author{
Laith Algharagholy ${ }^{\dagger a, b, d}$, Thomas Pope ${ }^{\dagger c}$, Qusiy Al-Galiby ${ }^{\dagger b, e}$, Hatef Sadeghi ${ }^{+b}$, Steve W.D. Bailey ${ }^{+b}$, \\ and Colin J. Lambert ${ }^{\text {tb* }}$
}

\begin{abstract}
We investigate the molecular sensing properties of carbon nanotube-boron nitride-carbon nanotube (CNT$\mathrm{BN}-\mathrm{CNT}$ ) junctions. We demonstrate that the electrical conductance of such a junction changes in response to the binding of an analyte molecule to the region of BN. The change in conductance depends on the length of the BN spacer and the position of the analyte and therefore we propose a method of statistically analysing conductance data. We demonstrate the ability to discriminate between analytes, by computing the conductance changes due to three analytes (benzene, thiolcapped oligoyne and a pyridyl-capped oligoyne) binding to junctions with five different lengths of BN spacer.
\end{abstract}

\section{Introduction}

Chemical sensors that work as electronic noses have attracted extensive attention, because they possess high sensitivity and selectivity towards target analytes, ranging from metal ions and anions to organic neutral chemicals and biological molecules ${ }^{1-3}$. Label-free methods for detecting small molecules are a desirable target technology, because they avoid the need for chemical modification or separation of the analytes, potentially leading to lower costs. One approach to developing such sensors involves measuring the electrical conductance of single-molecule junctions ${ }^{4-12}$. In principle such devices are capable of detecting a single analyte molecule, but controlling their junction separation and stability is difficult ${ }^{13-15}$ . Other techniques for molecular sensing ${ }^{16}$ involve measuring the change in electrical conductance of carbon nanotubes in response to molecular ${ }^{17}$ or changes in their vibrational ${ }^{18}$. In this paper, our aim is to build upon such approaches by demonstrating that single-molecule sensing capabilities can be significantly improved by utilising carbon/boron-nitride/carbon hetero nanotube junctions. Such nanotubes can be regarded as sculpturenes; ie novel nanometre-scale objects, obtained by

\footnotetext{
a. College of Computer Science and Mathematics, Al-Qadisiyah University, Diwaniyah, 58002, IRAQ.

b. Quantum Technology Centre, Lancaster University, Lancaster LA1 4YB, UK.

c. Physics Department, Catania University, 95123 Catania, Italy.

d. College of Basic Education, Sumer University, Al-Refayee, 64001, IRAQ

e. Physics Department, Al-Qadisiyah University, Diwaniyah, 58002, IRAQ

+ Authors contributed equally.

*Corresponding author: c.lambert@lancaster.ac.uk

Electronic Supplementary Information (ESI) available: [details of any supplementary information available should be included here]. See DOI: $10.1039 / \mathrm{x} 0 \mathrm{xx} 00000 \mathrm{x}$
}

cutting selected shapes from layered materials and allowing the shapes to reconstruct ${ }^{19-21}$. The simplest examples of sculpturenes are formed by cutting straight nanoribbons from bilayer graphene and allowing the edges to reconstruct to maximise $\mathrm{sp}^{2}$ bonding. If the width of the nanoribbon is sufficiently small (i.e. of order $3 \mathrm{~nm}$ or less) then the whole ribbon can reconstruct to form a carbon nanotube (CNT), with a pre-defined location and chirality. This cutting can be achieved using lithographic ${ }^{22-24}$, chemical ${ }^{25-28}$ or sonochemical $^{29,30}$ techniques. If the graphene layers are contacted with a boron-nitride $(\mathrm{BN})^{31}$, the reconstructed nanotube will be of a hetero structure. Previous studies have shown that the electronic properties and the stability of such hetero structures depend on the configuration of the $\mathrm{B}, \mathrm{N}$ and the $C$ atoms. It has been shown that doping an armchair CNT with a BN region leads to a tuneable HOMO-LUMO gap ${ }^{32,33}$. It is also known that the CNT-BN interface leads to localized states ${ }^{20,34-36}$. These states are present in doped nanotubes ${ }^{37-40}$ and similar interfaces in graphene ${ }^{31}$.

In what follows we investigate the sensing capabilities of a CNT-BN-CNT structure formed from two $(6,6)$ CNTs connected via an equivalent $(6,6) \mathrm{BN}$ nanotube. An electrical current flows through the BN from one CNT to the other and our aim is to understand the change in conductance of such a structure when a single analyte molecule binds to it. Since the BN possesses a large energy gap around the Fermi energy ${ }^{38,41-43}$, it behaves as an insulating barrier, which fixes the distance between the two CNT electrodes. We shall demonstrate that when an analyte binds to the $\mathrm{BN}$, the change in conductance depends on both the nature of the analyte and on the fixed length of the BN barrier layer. By analysing the response of 
devices with different BN-lengths, a unique fingerprint to each analyte is acquired which can be used for discrimination.

\section{Characterizing the junction}

Figure 1 shows five sculpturene junctions (labelled a-e) constructed from two $(6,6)$ armchair CNTs connected by BN barrier layers of lengths ranging from one to five unit cells. To construct these junctions, we relaxed hetero nanoribbons using the SIESTA implementation of DFT ${ }^{44}$ to minimise the forces on the atoms. In all

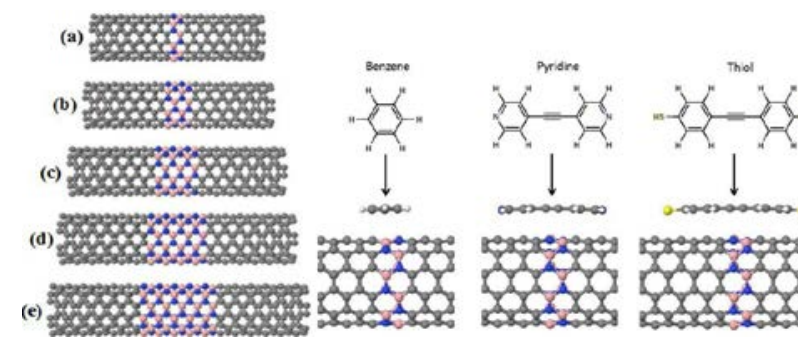

Fig 1: (Left) Hetero junctions constructed from two $(6,6)$ Carbon nanotubes joined by a BN insulating layer of varying length. (Right) an example of CNT-BN with three anylate molecules ( benzene, pyridine and thiol).

cases, we used the Ceperley-Alder (CA) exchange correlation functional, with norm-conserving pseudopotentials and double zeta polarized (DZP) basis sets of pseudo atomic orbitals.

The transmission coefficients, $T(E)$, for electrons of energy $E$ passing through the BN barrier are obtained using the Green's functionbased transport code GOLLUM ${ }^{45}$, which utilises the DFT-based hamiltonian from SIESTA. We show in figure 2 the transmission coefficients for each junction in isolation. When $E$ lies within the band gap of the $B N, T(E)$ decays exponentially with the length of the BN barrier as

$$
T(E)=T_{c} e^{-\beta x}
$$

where $\boldsymbol{T}_{\boldsymbol{c}}$ represents the effects of scattering at the BN-C interface and $\boldsymbol{x}$ is the barrier length. At $E=E_{F}$, where $E_{F}$ is the Fermi energy (ie Dirac point) of the CNTs, we calculate that the attenuation factor, $\boldsymbol{\beta}$ $=-3.6 \pm 0.2$ (see figure 3 ), which is of the same order as that of a typical oligoyne ${ }^{46}$.

The local density of states at the Fermi energy for the $1 \mathrm{BN}$ hetero junction was computed in Fig $\mathrm{S} 1$ of $\mathrm{SI}$, a state associated with the $\mathrm{N}$ $C$ interface can be seen and since this state bridges the junction, it is responsible for a small peaks in $T(E)$ near $E_{F}$. This can be seen most prominently in the transport curves (Fig S2 of $\mathrm{SI}$ ) for the $2 \mathrm{BN}$ and $3 B N$ junctions. Since the transport through the $1 B N$ junction is high irrespective of this effect, the peaks are not as clear. The only states near the Fermi energy localised on the BN buffer are these B-C and $\mathrm{N}-\mathrm{C}$ interface states. Therefore, for a clean junction, they represent the smallest distance between the two electrodes and any effect a molecule has on the junction will depend strongly on the molecule's interaction with these states.

\section{Discriminating single-molecule sensing of the device}

To test the sensing capability of each device, we placed analyte molecules at various locations in the vicinity of the BN spacer and computed the resulting transmission coefficient. Figure 2 shows results for the thiol-capped oligoyne. The dark lines show $T(E)$ for the clean junctions and the families of lighter lines show the $T(E)$ for various analyte binding

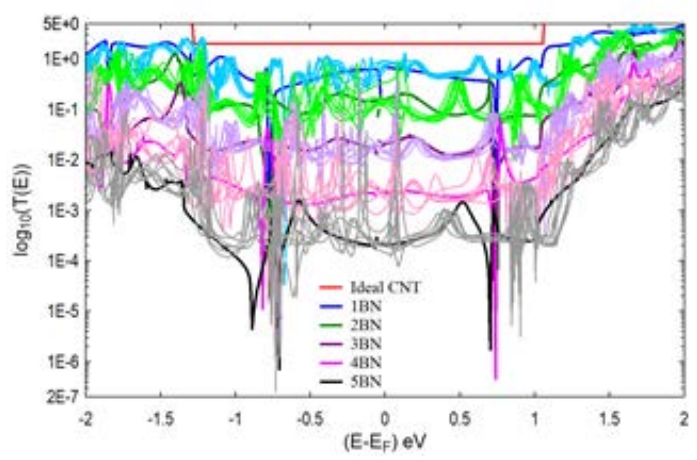

Fig 2: Plot of the logarithm of the transport of the thiolcapped oligoyne-doped junction, $\log _{10}(T(E))$, for $1 B N$ to $5 B N$. The darker lines represent the corresponding transport of the clean junction - also shown in Fig 1. For each junction, the thiol-capped oligoyne was placed at several locations.

locations. Results for two other analytes (pyridine-capped oligoyne and benzene) are shown in figures S5 and S6.

These figures show that the transmission coefficient fluctuates with different binding locations and therefore to sense and discriminate the analytes, a statistical approach to data analysis is needed.

In what follows, we define $T_{X, m}(E)$ to be the transmission coefficient in the presence of analyte $X$, (where $X=$ benzene, a pyridine-capped oligoyne (PY) or a thiol-capped oligoyne (SH)) located at position $m$. From the Landauer formula, the corresponding electrical conductance is $G_{X, m}\left(E_{F}\right)=G_{0} T_{X, m}\left(E_{F}\right)$. Therefore we define the quantity $\alpha_{X, m}\left(E_{F}\right)$ which is a measure of the difference between $G_{X, m}\left(E_{F}\right)$ and the conductance $G_{\text {bare }}\left(E_{F}\right)$ in the absence of a dopant ${ }^{47,48}$ :

$$
\alpha_{X, m}\left(E_{F}\right)=\log _{10} G_{X, m}\left(E_{F}\right)-\log _{10} G_{\text {bare }}\left(E_{F}\right)
$$

To differentiate between different junctions, we analyse the set of all values of $\alpha_{X, m}\left(E_{F}\right)$ for $E_{\min }<E_{F}<E_{\max }$ and configuration $m=$ $1, \ldots, M$ belonging to a given analyte $X$. These values can be obtained experimentally by using a third gate electrode to sweep through a range of Fermi energies. The probability distribution of the set $\left\{\alpha_{x, m}\left(E_{F}\right)\right\}$ for a given $X$ is then defined by: 


$$
P_{X}(\alpha)=\frac{1}{M\left(E_{\max }-E_{\min }\right)} \sum_{n=1}^{M} \int_{E_{\min }}^{E_{\max }} d E \delta\left(\alpha-\alpha_{X, m}(E)\right)
$$

where $\boldsymbol{\delta}\left(\boldsymbol{\alpha}-\boldsymbol{\alpha}_{\boldsymbol{X}, \boldsymbol{m}}(\boldsymbol{E})\right)$ is a Dirac delta function.

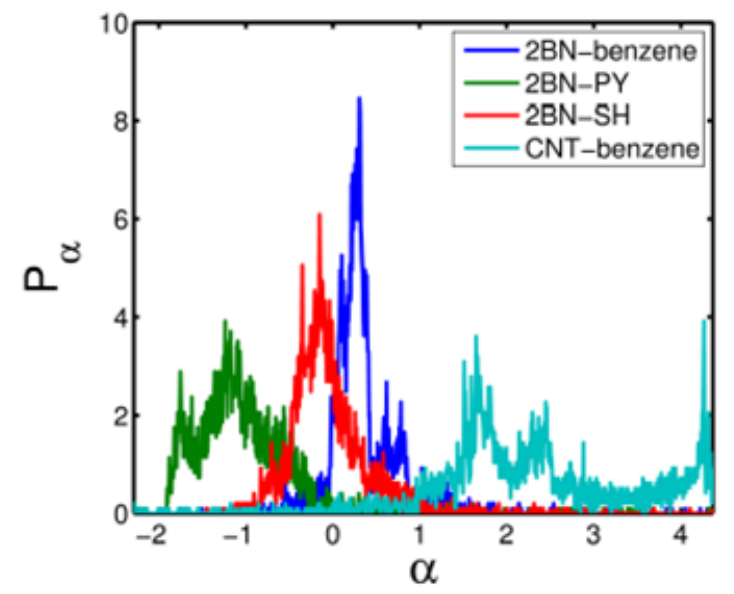

Fig 3: shows probability distribution $P_{x}(\alpha)$ of the set $\left\{\alpha_{x, m}(E)\right\}$ for a $2 \mathrm{BN}$ junction with benzene (blue), $2 \mathrm{BN}$ junction with $\mathrm{PY}$ (green), 2BN junction with SH (red) and ideal CNT with benzene (turquoise).

To demonstrate that a CNT-BN-CNT heterostructure is a more discriminating single-molecule sensor than a pure CNT, the magenta curve in Fig 3 shows the probability distribution $P_{X}(\alpha)$ for benzene adsorbed on a pure CNT while the blue curve shows the corresponding distribution for a hetero-tube containing a $2 \mathrm{BN}$ barrier. Clearly the latter is a more narrow distribution, which facilitates discriminating sensing. To demonstrate discrimination, the green and red curves in figure 3 show the distributions arising when Py-capped oligoynes and $\mathrm{SH}$-capped oligoynes bind to the $2 \mathrm{CN}$ hetero nanotube. These results for the three different analytes were obtained by sampling the curves at a uniformly spaced set of energies and creating histograms of the associated values of $\alpha_{x, m}(E)$. The calculations of the probability distributions for other heteronanotubes with different lengths of BN spacer are shown in Fig S7 of the SI.

\section{Conclusions}

Figure 3 shows that the different analytes lead to peaks in $P_{x}(\alpha)$ located at different values of $\alpha$ and therefore the $2 \mathrm{BN}$ structure is able to discriminate. This is in marked contrast with a pure CNT, which possesses rather broad peaks consequently a reduced the ability to discriminate. This broadening is illustrated in figure 3 which shows $P_{X}(\alpha)$ for benzene adsorbed on a $(6,6)$ CNT with no barrier. As well as showing a high sensitivity to the presence of a molecule, the junctions also show promising results suggesting a high level of selectivity. Since these junctions are reusable, they offer great versatility in probing the electronic properties of analytes. More importantly, the junctions are stable. In practice, the atomic-scale detail of hetero-junctions will not be known and will vary from sample to sample. Therefore it will be necessary to calibrate each junction for the range of analytes of interest and to preserve the calibration, the junctions must be stable. This feature of CNT-BN-CNT junctions makes them particularly attractive compared with single-molecule junctions, which usually are not stable over long periods of time.

\section{Acknowledgment}

This work is supported by the UK EPSRC, EP/K001507/1, EP/J014753/1, EP/H035818/1, and from the EU ITN MOLESCO 606728 and the Ministry of Higher Education and Scientific Research, Al Qadisiyah University and Thi-Qar University, IRAQ.

\section{References}

1. Schrader, T., Creative Chemical Sensor Systems. Springer: 2007; Vol. 277.

2. McFarland, A. D.; Van Duyne, R. P. Nano letters 2003, 3, (8), 1057-1062.

3. Al-Galiby, Q.; Grace, I.; Sadeghi, H.; Lambert, C. J. Journal of Materials Chemistry C 2015, 3, (9), 2101-2106.

4. Donhauser, Z.; Mantooth, B.; Kelly, K.; Bumm, L.; Monnell, J.; Stapleton, J.; Price, D.; Rawlett, A.; Allara, D.; Tour, J. Science 2001, 292, (5525), 2303-2307.

5. Andres, R. P.; Bein, T.; Dorogi, M.; Feng, S.; Henderson, J. I.; Kubiak, C. P.; Mahoney, W.; Osifchin, R. G.; Reifenberger, R. science 1996, 272, (5266), 1323-1325.

6. Gimzewski, J. K.; Joachim, C. science 1999, 283, (5408), 16831688.

7. Repp, J.; Meyer, G.; Paavilainen, S.; Olsson, F. E.; Persson, M. science 2006, 312, (5777), 1196-1199.

8. Wold, D. J.; Haag, R.; Rampi, M. A.; Frisbie, C. D. The Journal of Physical Chemistry B 2002, 106, (11), 2813-2816.

9. Fan, F.-R. F.; Yang, J.; Cai, L.; Price, D. W.; Dirk, S. M.; Kosynkin, D. V.; Yao, Y.; Rawlett, A. M.; Tour, J. M.; Bard, A. J. Journal of the American Chemical Society 2002, 124, (19), 5550-5560.

10. Xu, B.; Tao, N. J. science 2003, 301, (5637), 1221-1223.

11. Haiss, W.; van Zalinge, H.; Higgins, S. J.; Bethell, D.; Höbenreich, H.; Schiffrin, D. J.; Nichols, R. J. Journal of the American Chemical Society 2003, 125, (50), 15294-15295.

12. Li, Z.; Han, B.; Meszaros, G.; Pobelov, I.; Wandlowski, T.; Błaszczyk, A.; Mayor, M. Faraday Discussions 2006, 131, 121-143.

13. Cui, X.; Primak, A.; Zarate, X.; Tomfohr, J.; Sankey, O.; Moore, A.; Moore, T.; Gust, D.; Harris, G.; Lindsay, S. science 2001, 294, (5542), 571-574.

14. Zalinge, H. Physical Chemistry Chemical Physics 2004, 6, (17), 4330-4337.

15. Haiss, W.; Wang, C.; Grace, I.; Batsanov, A. S.; Schiffrin, D. J.; Higgins, S. J.; Bryce, M. R.; Lambert, C. J.; Nichols, R. J. Nature materials 2006, 5, (12), 995-1002.

16. Bachtold, A.; Hadley, P.; Nakanishi, T.; Dekker, C. Science 2001, 294, (5545), 1317-1320.

17. Besteman, K.; Lee, J.-O.; Wiertz, F. G.; Heering, H. A.; Dekker, C. Nano letters 2003, 3, (6), 727-730.

18. Chaste, J.; Eichler, A.; Moser, J.; Ceballos, G.; Rurali, R.; Bachtold, A. Nature nanotechnology 2012, 7, (5), 301-304.

19. Algharagholy, L.; Bailey, S. W.; Pope, T.; Lambert, C. J. Physical Review B 2012, 86, (7), 075427. 
20. Algharagholy, L.; Pope, T.; Bailey, S. W.; Lambert, C. J. New Journal of Physics 2014, 16, (1), 013060.

21. Qi, J. S.; Huang, J. Y.; Feng, J.; Shi, D. N.; Li, J. ACS nano 2011, 5, (5), 3475-3482.

22. Han, M. Y.; Özyilmaz, B.; Zhang, Y.; Kim, P. Physical review letters 2007, 98, (20), 206805.

23. Chen, Z.; Lin, Y.-M.; Rooks, M. J.; Avouris, P. Physica E: Lowdimensional Systems and Nanostructures 2007, 40, (2), 228-232.

24. Tapasztó, L.; Dobrik, G.; Lambin, P.; Biró, L. P. Nature nanotechnology 2008, 3, (7), 397-401.

25. Datta, S. S.; Strachan, D. R.; Khamis, S. M.; Johnson, A. C. Nano letters 2008, 8, (7), 1912-1915.

26. Ci, L.; Xu, Z.; Wang, L.; Gao, W.; Ding, F.; Kelly, K. F.; Yakobson, B. I.; Ajayan, P. M. Nano Research 2008, 1, (2), 116-122.

27. Campos, L. C.; Manfrinato, V. R.; Sanchez-Yamagishi, J. D.; Kong, J.; Jarillo-Herrero, P. Nano letters 2009, 9, (7), 2600-2604.

28. Campos-Delgado, J.; Romo-Herrera, J. M.; Jia, X.; Cullen, D. A.; Muramatsu, H.; Kim, Y. A.; Hayashi, T.; Ren, Z.; Smith, D. J.; Okuno, Y. Nano letters 2008, 8, (9), 2773-2778.

29. Li, X.; Wang, X.; Zhang, L.; Lee, S.; Dai, H. Science 2008, 319, (5867), 1229-1232.

30. Wu, Z.-S.; Ren, W.; Gao, L.; Liu, B.; Zhao, J.; Cheng, H.-M. Nano Research 2010, 3, (1), 16-22.

31. Liu, Z.; Ma, L.; Shi, G.; Zhou, W.; Gong, Y.; Lei, S.; Yang, X.; Zhang, J.; Yu, J.; Hackenberg, K. P. Nature nanotechnology 2013, 8, (2), 119-124.

32. Yin, Z.; Wu, S.; Zhou, X.; Huang, X.; Zhang, Q.; Boey, F.; Zhang, H. Small 2010, 6, (2), 307-312.

33. Terrones, M.; Romo-Herrera, J.; Cruz-Silva, E.; López-Urías, F.; Munoz-Sandoval, E.; Velázquez-Salazar, J.; Terrones, H.; Bando, Y.; Golberg, D. Materials Today 2007, 10, (5), 30-38.

34. Jiuxu, S.; Yintang, Y.; Hongxia, L.; Lixin, G. Journal of Semiconductors 2011, 32, (4), 042003.

35. Nguyen, H. D.; Ono, T. The Journal of Physical Chemistry C 2013, 117, (46), 24115-24120.

36. Meunier, V.; Roland, C.; Bernholc, J.; Nardelli, M. B. Applied Physics Letters 2002, 81, (1), 46-48.

37. Charlier, J.-C. Accounts of chemical research 2002, 35, (12), 1063-1069.

38. Golberg, D.; Bando, Y.; Huang, Y.; Terao, T.; Mitome, M.; Tang, C.; Zhi, C. Acs Nano 2010, 4, (6), 2979-2993.

39. Reyes, S. A.; Struck, A.; Eggert, S. Physical Review B 2009, 80, (7), 075115.

40. Peng, S.; Cho, K. Nano letters 2003, 3, (4), 513-517.

41. Zhi, C.; Bando, Y.; Tang, C.; Xie, R.; Sekiguchi, T.; Golberg, D. Journal of the American Chemical Society 2005, 127, (46), 1599615997.

42. Chopra, N. G.; Luyken, R.; Cherrey, K.; Crespi, V. H.; Cohen, M. L.; Louie, S. G.; Zettl, A. science 1995, 269, (5226), 966-967.

43. Soler, J. M.; Artacho, E.; Gale, J. D.; García, A.; Junquera, J.; Ordejón, P.; Sánchez-Portal, D. Journal of Physics: Condensed Matter 2002, 14, (11), 2745.

44. Rocha, A. R.; Garcia-Suarez, V. M.; Bailey, S. W.; Lambert, C. J.; Ferrer, J.; Sanvito, S. Nature materials 2005, 4, (4), 335-339.

45. Ferrer, J.; Lambert, C.; García-Suárez, V.; Manrique, D. Z.; Visontai, D.; Oroszlany, L.; Rodríguez-Ferradás, R.; Grace, I.; Bailey, S.; Gillemot, K. New Journal of Physics 2014, 16, (9), 093029.

46. Moreno-García, P.; Gulcur, M.; Manrique, D. Z.; Pope, T.; Hong, W.; Kaliginedi, V.; Huang, C.; Batsanov, A. S.; Bryce, M. R.; Lambert, C. Journal of the American Chemical Society 2013, 135, (33), 1222812240.
47. Sadeghi, H.; Algharagholy, L. A.; Pope, T.; Bailey, S. W.; Visontai, D.; Manrique, D. Z.; Ferrer, J.; García-Suárez, V. M.; Sangtarash, S.; Lambert, C. J. The Journal of Physical Chemistry B 2014.

48. Sadeghi, H.; Bailey, S.; Lambert, C. J. Applied Physics Letters 2014, 104, (10), 103104. 


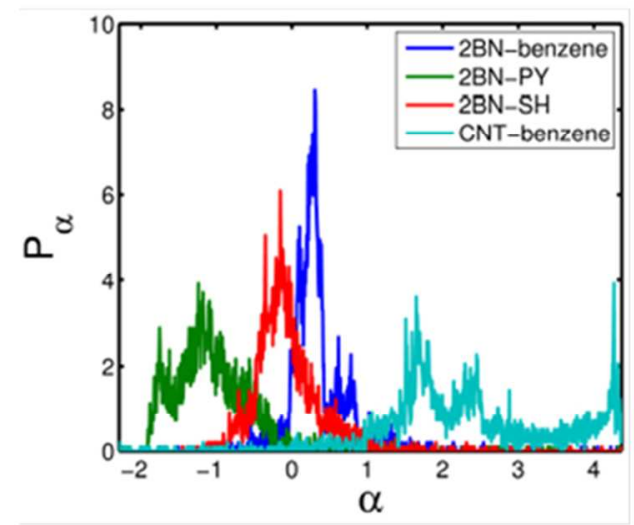

$254 \times 190 \mathrm{~mm}(96 \times 96$ DPI) 


\section{Supplementary Information}

\section{Sensing Single Molecules with Carbon-Boron-Nitride Nanotubes}

Laith Algharagholy ${ }^{\mathrm{a}, \mathrm{b}, \mathrm{d}}$, Thomas Pope ${ }^{\mathrm{c}}$, Qusiy Al-Galiby ${ }^{\mathrm{b}, \mathrm{e}}$,Hatef Sadeghi ${ }^{\mathrm{b}}$, Steve W.D. Bailey ${ }^{\mathrm{b}}$, and Colin J. Lambert ${ }^{\mathrm{b}^{*}}$

${ }^{\text {a }}$ College of Computer Science and Mathematics, Al-Qadisiyah University, Diwaniyah, Iraq

b Quantum Technology Centre, Lancaster University, Lancaster LA1 4YB, UK

c Physics Department, Catania University, 95123 Catania, Italy

${ }^{\mathbf{d} C o l l e g e ~ o f ~ B a s i c ~ E d u c a t i o n, ~ S u m e r ~ U n i v e r s i t y, ~ A l-R e f a y e e, ~ D h i-Q a r, ~ I r a q ~}$

${ }^{\text {e}}$ Physics Department ,Al-Qadisiyah University, Diwaniyah, Iraq

${ }^{\dagger}$ Authors contributed.

*Corresponding author: c.lambert@lancaster.ac.uk

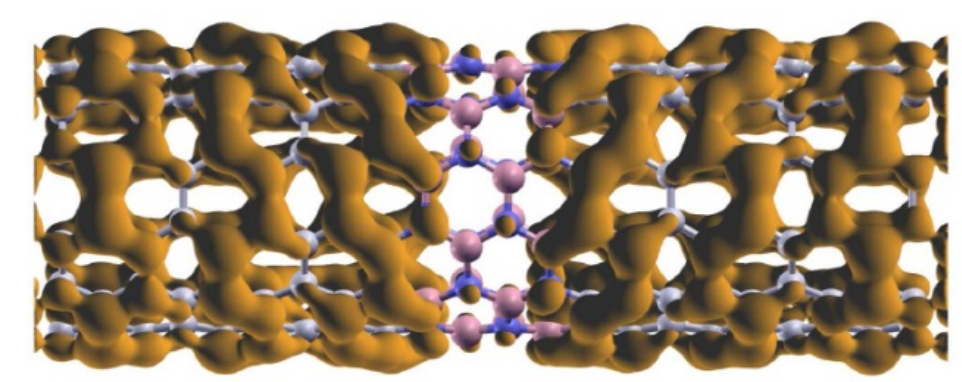

Fig S1: Local Density of States for the energy range $E_{F}-0.01 \rightarrow E_{F}+0.01$ for the $1 B N$ junction

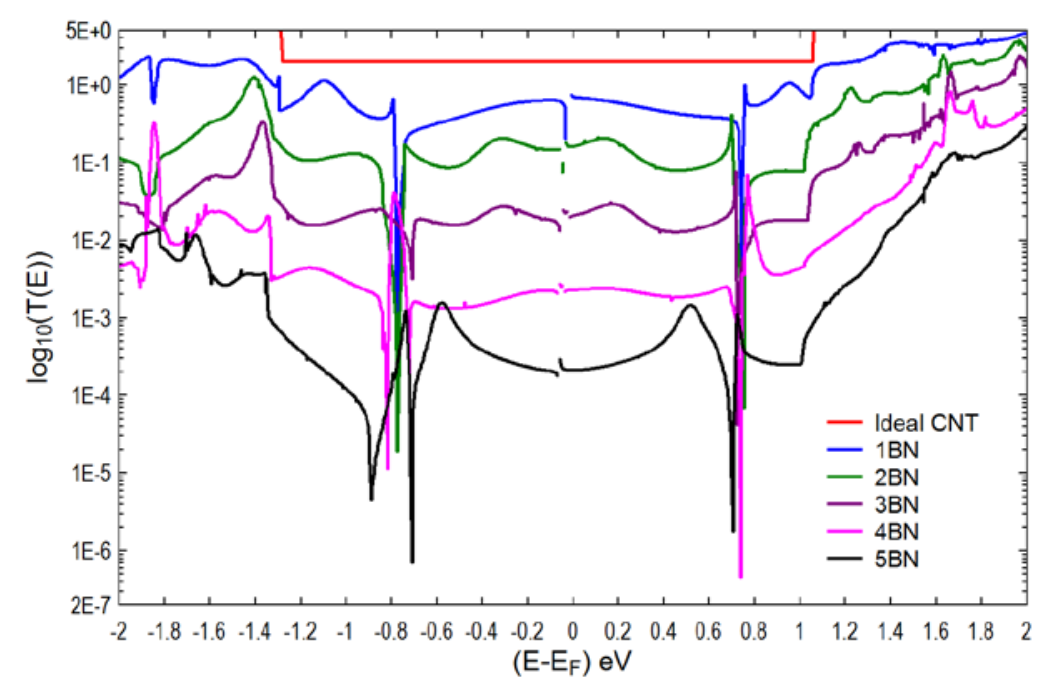

Fig S2: Transport curves for junctions a-e of Figure 1, which compare with an Ideal CNT (line in red). 


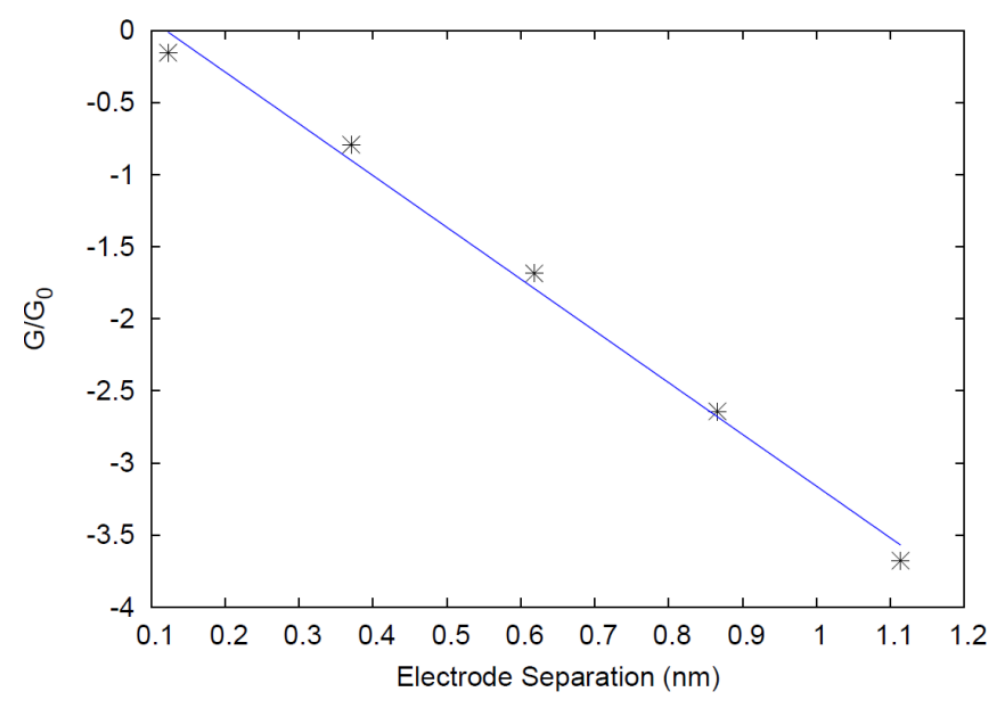

Fig S3: Conductance of the junction as a function of electrode separation.

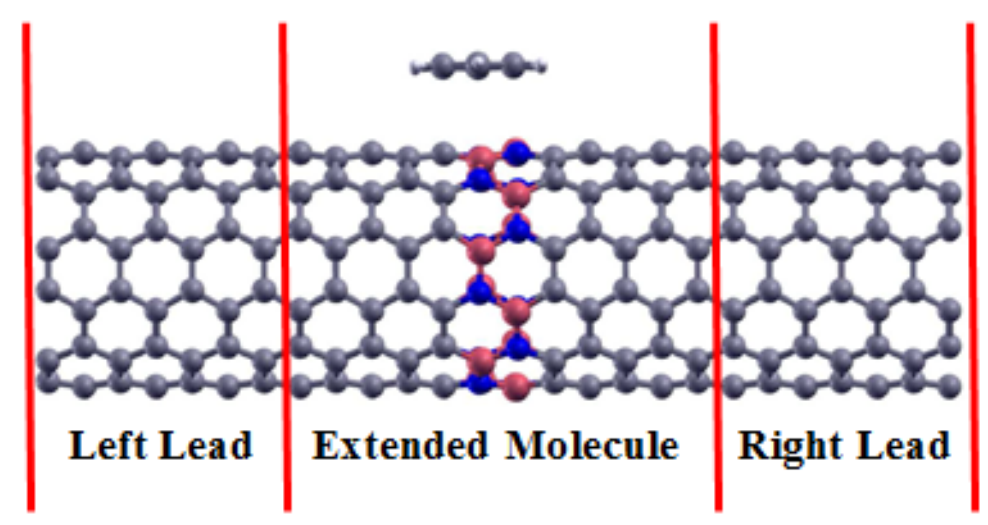

Fig S4: Example of the benzene-doped junction. 


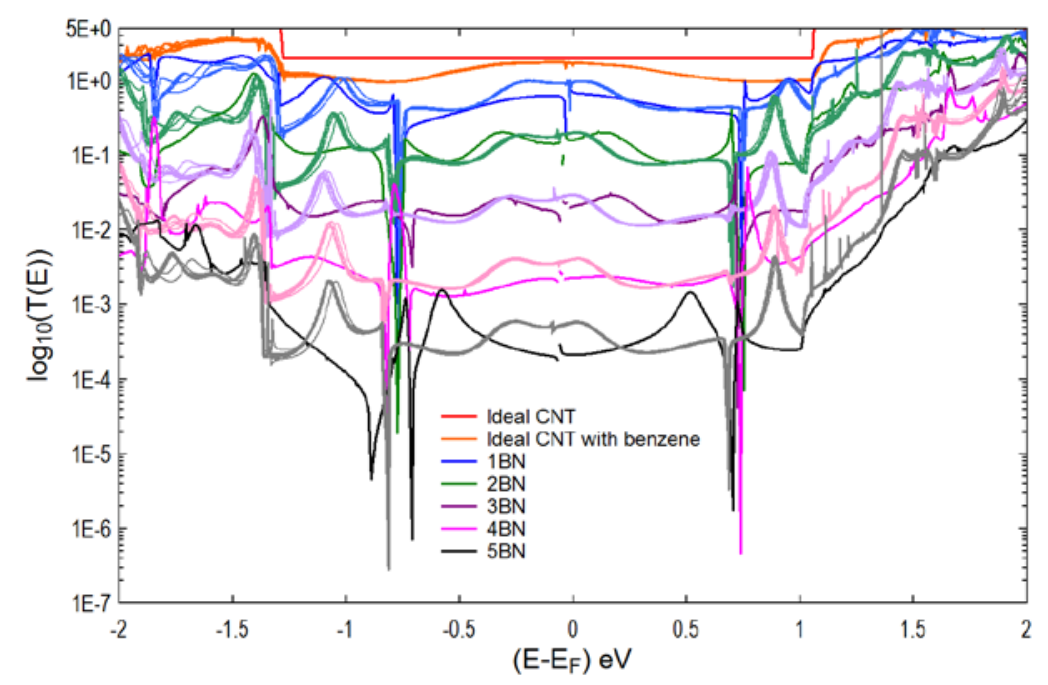

Fig S5: Plot of the logarithm of the transport of the benzene-doped junction, $\log _{10}(T(E))$, for different junction sizes $(1 B N-5 B N)$. The darker lines represent the corresponding transport of the clean junctionalso shown in figure 1. For each junction, the benzene rings were placed at several locations. 


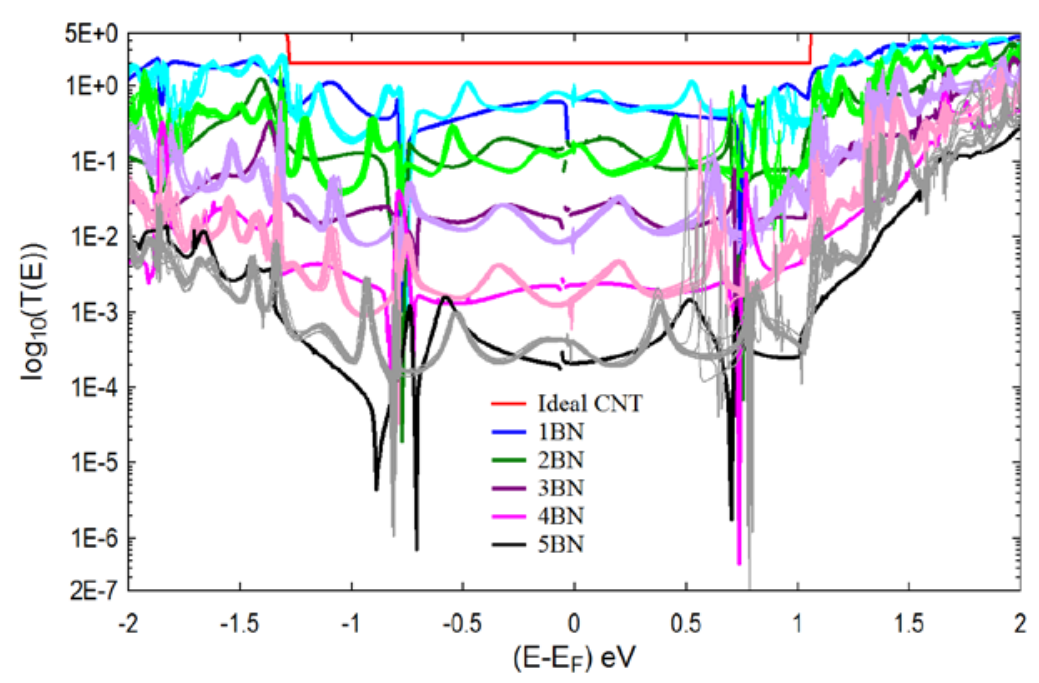

Fig S6: Plot of the logarithm of the transport of the pyridine-capped oligoyne-doped junction, $\log _{10}(T(E))$, for $1 B N$ to $5 B N$. The darker lines represent the corresponding transport of the clean junction - also shown in Fig 1. For each junction, the pyridine-capped oligoyne was placed at several locations. 

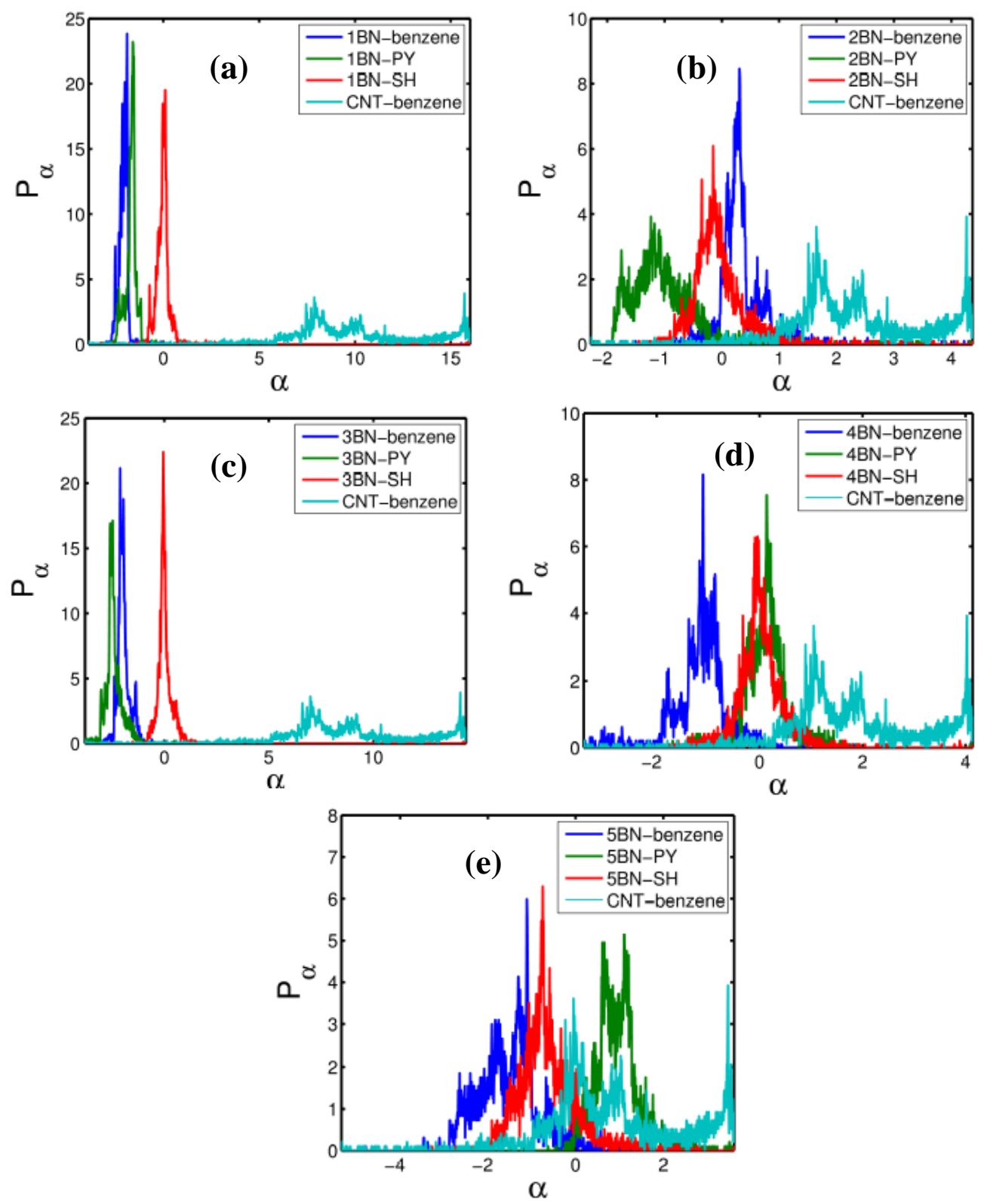

Fig S7: The probability distribution $P_{X}(\alpha)$ of the set $\left\{\alpha_{X, m}(E)\right\}$ for (a) $1 B N$ junction with benzene (blue), $1 B N$ junction with PY (green), 1BN junction with SH (red) and ideal CNT with benzene (turquoise). (b) 2BN junction with benzene (blue), 2BN junction with PY (green), 2BN junction with SH (red) and ideal CNT with benzene (turquoise). (c) 3BN junction with benzene (blue), 3BN junction with PY (green), 3BN junction with SH (red) and ideal CNT with benzene (turquoise). (d) 4BN junction with benzene (blue), 4BN junction with $P Y$ (green), $4 B N$ junction with $S H$ (red) and ideal CNT with benzene (turquoise). (e) 5BN junction with benzene (blue), 5BN junction with PY (green), 5BN junction with $S H$ (red) and ideal CNT with benzene (turquoise). 